\title{
QLad: A Noise-Free Quantum Memory for Broadband Light at Room Temperature
}

\author{
K. T. Kaczmarek ${ }^{1}$, P. M. Ledingham ${ }^{1}$, B. Brecht ${ }^{1}$, A. Feizpour ${ }^{1}$, G. S. Thekkadath ${ }^{2}$, \\ S. E. Thomas ${ }^{1}$, J. H. D. Munns ${ }^{1}$, D. J. Saunders ${ }^{1}$, I. A. Walmsley ${ }^{1}$, J. Nunn ${ }^{1}$ \\ ${ }^{1}$ Clarendon Laboratory, University of Oxford, Oxford, OX1 3PU, UK \\ ${ }^{2}$ National Research Council of Canada, Ottawa, K1N 5A2, Canada \\ j.nunn1@physics.ox.ac.uk
}

\begin{abstract}
We implement a low-noise, broadband quantum memory for light via off-resonant two-photon absorption in warm atomic vapour. We store heralded single photons and verify that the retrieved fields are anti-bunched.
\end{abstract}

(C) 2016 Optical Society of America

OCIS codes: (270.5585) Quantum information and processing, (210.4680) Optical memories.

\section{Introduction}

Linear optical logic elements are non-deterministic, and a multiplexing strategy is required to actively pick out successful gate operations. Quantum memories promise scalability via temporal multiplexing, where successful outputs are stored until all parts of a photonic circuit have executed [1].

In this work, we propose and demonstrate a new memory protocol - the quantum ladder (QLad) — based on off-resonant two-photon absorption in a warm atomic vapour.

\section{QLad protocol}

Atomic vapour quantum memories are typically based on Raman storage or electromagnetically induced transparency (EIT), in which a strong control field mediates storage of an incident signal into a spin-wave coherence in the ground state hyperfine manifold. The spin-wave can survive for a long time $(\mu \mathrm{s}-\mathrm{ms})$, which is needed for quantum communications applications where the memory lifetime should be long compared to the signal latency over the channel length. However, storage in the ground state manifold comes with several difficulties. First, the storage state must be emptied by optical pumping. Second, the fidelity of storage in such memories is limited by four-wave mixing, in which the storage state is populated by spurious excitations generated through spontaneous Raman scattering [2,3]. Third, it is challenging to separate the bright control field from the weak retrieved signal, as they are separated by a small frequency difference $(\sim \mathrm{GHz})$.

The QLad protocol takes its cue from the observation that for local photonic synchronisation applications, the memory lifetime can be much shorter, provided that it is long compared to the clock cycle of the system. Running at $1 \mathrm{GHz}$, a storage time of several tens of ns is sufficient to synchronise over many clock cycles. Therefore one may consider light storage in the excited states of an atomic vapour [4]. As shown in the figure, we consider an atomic vapour with a 'ladder' of near-degenerate optical transitions. An incident signal pulse, detuned from the lower resonance, is stored by a counter-propagating control pulse that stimulates two-photon absorption into the uppermost state.

Since the storage state is a doubly-excited orbital state, it is empty at room temperature and no optical pumping is needed to initialise the memory. Furthermore, there is no equivalent noise process to the four-wave mixing noise described above for ground-state memories: the storage state cannot be populated through spontaneous emission. Finally, the counterpropagating configuration allows for straightforward low-loss separation of the signal and control, and it is Doppler-free when the two transitions are degenerate (when they have the same wavelength), meaning that the storage time can be as long as the fluorescence lifetime of the storage state, even in a hot vapour.

\section{Experiment}

We implemented a proof-of-principle experiment using warm Cs vapour. For technical convenience we chose the $6 S_{1 / 2} \rightarrow 6 P_{3 / 2} \rightarrow 6 D_{5 / 2}$ ladder. These transitions are not close to degeneracy, so the Doppler cancelation alluded to 
(a)

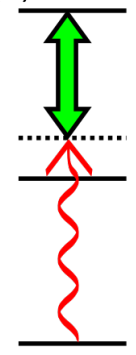

\section{(b)}
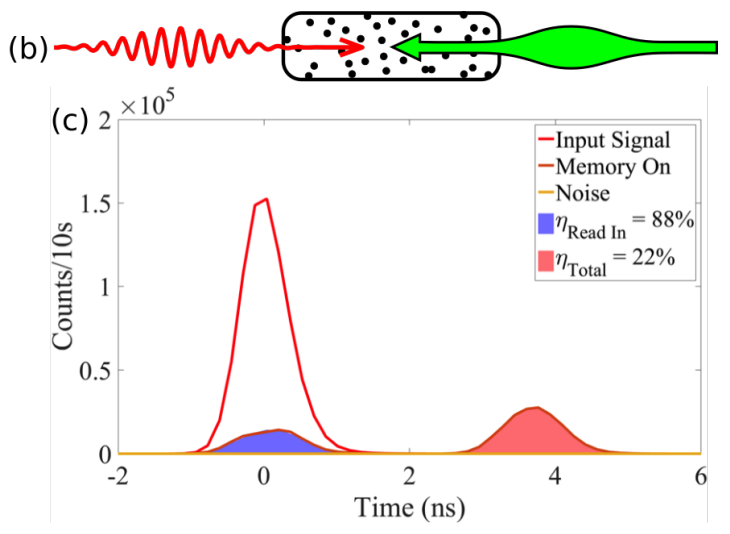

Fig. 1. (a) The near-degenerate ladder-type energy level configuration used for QLad storage, showing the off-resonant two-photon absorption mediated by the control field. (b) Signal and control fields impinge on a warm vapour from opposite directions in a Doppler-free configuration. (c) Experimental demonstration of storage and retrieval of weak coherent states.

above is only partial, and the storage time is limited to $\sim 5 \mathrm{~ns}$. But this does not affect the kinematics of the off-resonant storage and retrieval interactions, and we therefore use this system to explore the efficiency and noise characteristics of QLad and to verify quantum-limited operation.

The time-series in part (c) of the figure shows the storage and retrieval of weak coherent states with mean photon number $\sim 0.4$. While the storage efficiency is high (nearly $90 \%$ ), the retrieval efficiency is limited by Doppler dephasing. Crucially though, the noise level is extremely low, and in fact we could not detect any added noise above the dark counts of our detectors.

We have also tested the QLad memory by storing and retrieving GHz-bandwidth heralded single photons generated by parametric downconversion in a periodically-poled KTP waveguide [3]. We directed the fields retrieved from the memory into a Hanbury-Brown-Twiss interferometer and measured the normalised autocorrelation to be $g^{(2)}=0.39 \pm$ 0.05 , which establishes that the memory preserves the anti-bunched character of the single-photon input.

\section{Summary and Outlook}

We have described the QLad memory protocol, which combines the advantageous features of several other protocols in order to achieve noise-free, broadband, room-temperature light storage in a technically simple set-up that allows for efficient separation of the signal and control fields. The price for these advantages is the relatively short storage time that can be achieved in excited states. However, long storage times are not required for local synchronisation applications at high clock rates. In future, QLad storage may be combined with further manipulations to map stored excitations into ground state or Rydberg coherences, providing a route to extended lifetimes or giant non-linearities.

\section{References}

1. J. Nunn, N. Langford, W. Kolthammer, T. Champion, M. Sprague, P. Michelberger, X.-M. Jin, D. England, and I. Walmsley, "Enhancing multiphoton rates with quantum memories," Physical review letters 110, 133,601 (2013).

2. N. Lauk, C. O'Brien, and M. Fleischhauer, "Fidelity of photon propagation in electromagnetically induced transparency in the presence of four-wave mixing," Physical Review A 88, 013,823 (2013).

3. P. Michelberger, T. Champion, M. Sprague, K. Kaczmarek, M. Barbieri, X. Jin, D. England, W. Kolthammer, D. Saunders, J. Nunn et al., "Interfacing ghz-bandwidth heralded single photons with a room-temperature raman quantum memory," arXiv preprint arXiv:1405.1470 (2014).

4. L. Li and A. Kuzmich, "Quantum memory with strong and controllable rydberg-level interactions," Nature Communications 7 (2016). 